Comment. Math. Helv. 74 (1999) 1-21

0010-2571/99/010001-21 \$1.50+0.20/0
(C) 1999 Birkhäuser Verlag, Basel

Commentarii Mathematici Helvetici

\title{
Geodesic foliations in Lorentz 3-manifolds
}

\author{
Abdelghani Zeghib
}

\begin{abstract}
We study geodesic foliations on manifolds endowed with Lorentz metrics. The (local) theory works formally exactly as in the Riemannian case, if the induced metric on the leaves is non-degenerate. We consider here some local and global properties in the degenerate case.
\end{abstract}

Mathematics Subject Classification (1991). 53B30, 57R30.

Keywords. Geodesic, lightlike, normal foliation.

\section{Introduction}

A Lorentz metric on a manifold $M$ is a (smooth) symmetric tensor of degree 2 and index 1 , that is a quadratic form of signature $-+\ldots+$, on the tangent space at each point of $M$.

Lorentz metrics give rise to the same tensorial and differential calculus as Riemannian metrics, e.g. Levi-Civita connections and so geodesics...

Nevertheless, there exist some deep differences between Riemannian and Lorentz geometry. For example, a Lorentz metric does not generate a structure of "standard" metric space. On the other hand, a Lorentz metric determines causal characters for tangent vectors and subspaces. To define them, let us denote the Lorentz metric by $<>$. A tangent vector $u$ (resp. a tangent subspace $E$ ) is spacelike if $\langle u, u\rangle$ is positive (resp. the restriction of $\langle>$ to $E$ is a positive definite scalar product), timelike if $\langle u, u\rangle$ is negative (resp. the restriction of $\langle>$ to $E$ is a Lorentzian scalar product, i.e. of index 1), and finally lightlike (or degenerate) if $\langle u, u\rangle=0$ (resp. the restriction of $\langle>$ to $E$ is a positive non-definite scalar product) (a lightlike vector is also called isotropic). Notice that $u$ is lightlike if and only if $u \in u^{\perp}$, in which case the metric on $u^{\perp}$ is degenerate (and positive) with nullity space exactly $\mathbf{R} u$.

Lightlike submanifolds. The geometry of submanifolds in Lorentz manifolds may be developed as in the Riemannian case, if we restrict ourselves (as in classical books in the field: [B-E], [O'N]...) to those with (everywhere) non-degenerate 
tangent spaces. In particular, induced metrics and connections are coherently defined in this case.

Our goal here is to look for (a particular type of) lightlike submanifolds. Let $V$ be a submanifold in a Lorentz manifold $(M,<>$ ), we call it lightlike (or degenerate) if for any $x \in V$, the tangent space $T_{x} V$ is a lightlike space. The nullity space of the restriction of the Lorentz metric to $V$ determines a field of directions that we call the normal direction of $V$. We denote it, as well as the 1-dimensional foliation that it generates by $\mathcal{N}$, and generally by $N$ a vector field tangent to it.

Furthermore, a lightlike submanifold possesses a degenerate Riemannian metric, which in the general case means the following: A degenerate Riemannian metric on a vector bundle $E$ over a manifold $V$ is a field of positive (but non necessarily definite) quadratic forms on it. In our case, the degenerate Riemannian metrics will have index of nullity 1 , and so they are the nicest after the Riemannian metrics.

\subsection{Geodesibility criterion. Geodesic foliations}

A submanifold $V$ (degenerate or not) is geodesic if it contains the geodesics of $M$ which are somewhere tangent to it (if $x(t)$ is a geodesic of $M$ defined in a neighborhood of 0 and $x^{\prime}(0) \in T_{x(0)} V$, then $x(t) \in V$ for $\mathrm{t}$ in a neighborhood of 0 .) This is equivalent to saying that, the space of $C^{1}$ vector fields on $V$ is invariant by covariant derivativion (this equivalence is valid for all torsion free connections $[\mathrm{K}-\mathrm{N}])$. Therefore, although there is no way to induce connections on lightlike submanifolds, the geodesic ones have such a connection. This connection is compatible with the degenerate Riemannian metric but not derived from it, as degenerate Riemannian metrics do not have Levi-Civita connections.

The following is a key fact:

Criterion 1. Let $V$ be a lightlike submanifold and $\mathcal{N}$ its 1 - dimensional normal foliation. If $V$ is geodesic, then $\mathcal{N}$ is (transversally) Riemannian in the following precise sense: any (local) flow generated by a vector field tangent to $\mathcal{N}$ preserves the induced degenerate Riemannian metric (note that it suffices to verify this for one such vector field).

Conversely, in codimension 1, a lightlike hypersurface having a transversally Riemannian normal foliation, is geodesic.

Furthermore, in any codimension, if $V$ is geodesic, the leaves of its normal foliation $\mathcal{N}$ are lightlike geodesic (in $M$ ).

Proof. Let $\nabla$ denote the Levi-Civita connection of $M$, and $N$ a local field tangent to the normal foliation of $V$.

The flow of $N$ preserves the degenerate Riemannian metric, iff, for any vector field $X$ tangent to $V$ and commuting with $N$ (i.e. preserved by the flow of $N$ ), the scalar product $\langle X, X\rangle$ is constant along the $N$-orbits, that is, $N<X, X\rangle=0$. 
We have: $N<X, X>=2<\nabla_{N} X, X>=2<\nabla_{X} N, X>$, since $[N, X]=0$. But: $\left.\left\langle\nabla_{X} N, X>=X<N, X>-<N, \nabla_{X} X\right\rangle=-<N, \nabla_{X} X\right\rangle$ (since $X$ is tangent to $V$, and thus by definition, $<N, X>=0$ ).

Now, if $V$ is geodesic, $\nabla_{X} X$ is tangent to $V$, and so $\left\langle N, \nabla_{X} X\right\rangle=0$. Therefore $N<X, X>=0$, and thus the flow of $N$ preserves $<,>$.

In codimension 1, the tangent space of $V$ is exactly the orthogonal of $N$. Therefore, by the calculation above, if the flow of $N$ preserves $\langle$,$\rangle , then \left\langle N, \nabla_{X} X\right\rangle=$ 0 , and hence $\nabla_{X} X$ is tangent to $V$, that is, $V$ is geodesic.

The last point is obvious, since a geodesic which is tangent somewhere to $\mathcal{N}$ must be lightlike and contained in $V$. But the lightlike curves of a lightlike submanifold are exactly the leaves of its normal foliation.

Interpretation. Let $\mathcal{F}$ be a codimension 1 foliation of a Riemannian manifold, and $\mathcal{N}$ its orthogonal 1-dimensional foliation. It is known that $\mathcal{F}$ has geodesic leaves (all of them), if and only if $\mathcal{N}$ is a Riemannian foliation (see for instance [Gh1]). This fact extends to Lorentz (or pseudo-Riemannian) manifolds, in a straightforward way, for foliations with non-lightlike leaves (i.e. $\mathcal{N}$ is transversal to $\mathcal{F}$ ). The proposition above, which deals with just a submanifold instead of a foliation, may be seen as a limit case of this fact. This leads us to consider:

Lightlike geodesic foliations. They are foliations with lightlike geodesic leaves. There is no natural way to go from a leaf to another, and the condition on a lightlike geodesic foliation is just the tangential one on its individual leaves, as in the proposition above. For a foliation $\mathcal{F}$ by lightlike leaves, we call the normal foliation of $\mathcal{F}$ (which is a sub-foliation of it), the 1 dimensional foliation obtained from the normal foliations of its leaves.

Hypothesis. To simplify, we assume all things (manifolds, submanifolds, foliations, sub-foliations...) orientable.

Proposition 2. Let $\mathcal{F}$ be a codimension 1 lightlike foliation of a Lorentz manifold $M$. Then $\mathcal{F}$ is geodesic if and only if its normal foliation $\mathcal{N}$ is leafwise transversally Riemannian, that is, any parameterization of $\mathcal{N}$ preserves the degenerate Riemannian metric on $T \mathcal{F}$.

On the other hand, a given $C^{k}$ foliation $\mathcal{F}$ is lightlike geodesible, i.e. lightlike geodesic for some $C^{k}$ Lorentz metric on $M$, if there is a 1 dimensional sub-foliation $\mathcal{N}$, of class $C^{k}$, which is leafwise Riemannian for some $C^{k}$ degenerate Riemannian metric on $T \mathcal{F}$.

Proof. The first part is straightforward from Criterion 1. For the second part, denote by $g$ the $C^{k}$ degenerate Riemannian metric on $T \mathcal{F}$, invariant by $\mathcal{N}$. From Criterion 1, it suffices to show that $g$ can be extended to a Lorentz metric $<$, > on $T M$. To get this, let $N$ be a non-singular vector field tangent to $\mathcal{N}, Z$ a vector 
field transverse to $\mathcal{F}$ (such a field exists by our orientability hypothesis), and $\mathcal{N}^{\prime}$ a complement space of $\mathcal{N}$ in $T \mathcal{F}$. We extend the metric so that: $Z$ is orthogonal to $\mathcal{N}^{\prime},\langle Z, N\rangle=1$ and we choose $\langle Z, Z\rangle$ arbitrarily (for example 0 ). It is straightforward to see that this gives a Lorentz metric.

\subsection{Some motivations}

Lightlike geodesic foliations may be seen as a tool as well as a (limit) structure:

Foliated geometric structures. It is a common principle to think of a "geometric structure" on the leaves of a compact foliated manifold as a structure (of the same type) on a single compact manifold. Following this idea, one may hope that some theorems have foliated versions. Many works by many authors confirm this point of view. We mention a few examples: a generalization by Zimmer of Mostow rigidity to foliations by hyperbolic manifolds [Zim]; a generalization by S. Adams and A. Freire of the Splitting theorem of Cheeger and Gromoll to foliations by non-negatively Ricci curved manifolds $[\mathrm{A}-\mathrm{F}]$; the theory of the foliated Plateau problem by Gromov [Gr1]; and finally Candel's Theorem on uniformization of surface laminations [Can]. The proposition above deals with leafwise Riemannian foliations. One may ask, which parts of Molino's structure Theorem, in particular about closures of leaves, may have a foliated version? One also may ask if there is a foliated version of Carrière's work on Riemannian flows [Mol].

Lorentz dynamics. We will discuss later the lightlike geodesibility of weak stable foliations of Anosov flows. It turns out that lightlike geodesic foliations appear in a more general setting, as "approximately stable foliations" associated to flows preserving Lorentz metrics. Indeed, lightlike geodesic foliations exist on a compact Lorentz manifold, provided it has a non-compact isometry group, see [D-G] and [Ze2].

Relativity and the global theory of foliations. A non-singular vector field $N$ on $M$ is lightlike (or isotropic, null, optical...) if $N(x)$ is isotropic, for all $x \in M$. The quotient bundle $N^{\perp} / N$ then inherits a Riemannian metric.

Observe that lightlike vector fields depend only on the conformal structure of $M$, and that a Lorentz conformal change on $M$ induces a Riemannian conformal change on $N^{\perp} / N$.

We will discuss in what follows some "optical" notions, which seem to be very important in relativity. For instance, they occupy a chapter, entitled "null congruences" in the book [P-R], where it is said that they "play an important part in electromagnetic and gravitational radiation theory and in the construction of exact solutions of Einstein's equations". Here we follow [R-T].

An optical geometry consists of giving a flag like $\left(N, N^{\perp}\right)$ together with a conformal Riemannian structure on $N^{\perp} / N$. An optical geometry determines a class 
of Lorentz conformal structures adapted to it.

A ray geometry is an optical geometry such that $N$ has geodesic orbits. It is remarkable that this condition doesn't depend on the adapted Lorentz structure.

A ray geometry is called without twist (resp. without shear) if $N^{\perp}$ is integrable (resp. if the flow of $N$ preserves the conformal structure on $N^{\perp} / N$ ).

So, in the twist and shear free case, we obtain a codimension 1 lightlike foliation, whose normal foliation preserves its "conformal degenerate Riemannian structure". This is slightly weaker than the fact that the foliation being geodesic. More precisely, this means that the foliation is umbilic. This motivates the study of geodesic foliations as a first step in studying the umbilic ones.

Electromagnetism is a structure which may enrich a ray geometry. Recall that a (sourceless) electromagnetic field on a Lorentz manifold is a harmonic 2-form, that is a 2-form $F$ such that $d F=d(* F)=0$, where $* F$ is the Hodge dual of $F$. This is a conformal notion in dimension 4 .

An electromagnetic field is adapted to a ray geometry, if both $F$ and $* F$ are annullated by $N$, that is $i_{N} F=i_{N} * F=0$ ( $N$ is called a characteristic vector field for the system $\{F, * F\}$ ).

There is a result due to I. Robinson, which asserts that a ray geometry has a non trivial adapted electromagnetic field, iff, it is shear free (see [R-T]). It was then observed that such a result requires analyticity of data in the general case. But, from a topological point of view, one may also observe that this is only a local result. It seems interesting to translate and consider these questions in the global theory of foliations. For example, when does a lightlike geodesic foliation possess a global adapted non trivial electromagnetic field?

In fact, conversely, one may go from electromagnetism to ray geometry. Indeed, given an electromagnetic field $F$, a characteristic vector field $N$ of the electromagnetic structure must be geodesic lightlike, and then determines a shear free ray geometry, for which $F$ is an adapted electromagnetic field (all this in dimension 4). Such a vector field $N$ exists, when the system of 2 -forms $\{F, * F\}$ has minimal rank. The electromagnetic field is then called singular or a pure radiation [Lic]. In classical terminology, a pure radiation occurs when the electric field strength and the magnetic field strength are orthogonal.

Summarizing, one may meet lightlike geodesic foliations, as associated to special (but omnipresent in exact solutions) electromagnetic fields.

\subsection{Constructions. Examples}

Let us now give some examples of lightlike geodesible foliations.

Suspensions. Let $\mathcal{L}$ be a foliation of a Riemannian manifold $M^{\prime}$, and $f$ a diffeomorphism of $M^{\prime}$ preserving $\mathcal{L}$ and also the restriction of the Riemannian metric to the leaves of $\mathcal{L}$.

Let $M=M^{\prime} \times[0,1] /{ }_{(x, 1) \sim(f(x), 0)}$ be the suspension of $f$. Let $\mathcal{N}$ be the fo- 
liation on $M$ determined by $[0,1]$, i.e. the orbits of the suspension flow, and $\mathcal{F}$ the saturation of $\mathcal{L}$ by $\mathcal{N}$. Then $\mathcal{F}$ is lightlike geodesible, with $\mathcal{N}$ as a normal foliation. (Warning: this construction generalizes that of suspension flows (from diffeomorphisms), but has nothing to do with foliations obtained by suspension of fundamental group actions on $S^{1}$ (see below)). Notice also that the Lorentz metric on $M$ is by no means a product of Lorentz or Riemannian metrics on $M^{\prime}$ and $[0,1]$.

Actions of Lie groups. Let $G$ be a Lie group acting locally freely with codimension 1 orbits on a manifold $M$. Assume that $G$ has a normal connected subgroup $N$ of dimension one. Denote by $\mathcal{F}$ the orbit foliation of $G$ and $\mathcal{N}$ the sub-foliation corresponding to $N$.

Proposition 3. $\mathcal{F}$ is lightlike geodesible with $\mathcal{N}$ as normal foliation.

Proof. Let $\bar{X}_{0}, \ldots, \bar{X}_{d}$ be a basis of the Lie algebra of $G$, with $\bar{X}_{0}$ corresponding to $N$. They determine fundamental vector fields $X_{0}, \ldots, X_{d}$ on $M$, which generate $T \mathcal{F}$. Consider on $T \mathcal{F}$ the degenerate Riemannian metric: $\left\langle X_{0}, X_{i}\right\rangle=0$ for all $i$, and $\left\langle X_{i}, X_{j}\right\rangle=\delta_{i j}$ for $i \neq 0$ and $j \neq 0$. Since $N$ is normal, any bracket $\left[X_{0}, X_{i}\right]$ is a multiple of $X_{0}$, and so the flow of $X_{0}$, i.e. the action of $N$ maps $X_{i}$ to a vector field of the form $X_{i}+f X_{0}$, for some function $f$. In particular $N$ preserves the degenerate Riemannian metric. The claim follows from Proposition 2.

As examples of groups admitting normal subgroups of dimension 1, we have all nilpotent groups: take any subgroup of dimension 1 in the center. There are also a lot of solvable groups which have such subgroups. The simplest of them is $A G$, the group of affine transformations of the line (see below).

\section{Rigidity results in dimension 3}

In dimension 3, the above suspension construction must start with a foliation of the 2-torus (we assumed all things orientable). Moreover, the gluing map must preserve a parameterization of that foliation. Hence, the obtained suspension foliation is defined by an action of $\mathbf{R}^{2}$.

On the other hand, in dimension 3 , the only groups acting locally freely with codimension one orbits are $\mathbf{R}^{2}$ or $A G$. Both of them as stated above give lightlike geodesible foliations.

The notion of lightlike geodesible foliations appears as a "geometrical" unification of locally free actions of Lie groups on 3-manifolds (although, dynamically, actions of $\mathbf{R}^{2}$ and $A G$ are very different). In fact, conversely, we dare ask:

Question. Is (up to finite covers) a codimension one lightlike geodesic foliation 
on a closed 3-manifold determined by a locally free action of $\mathbf{R}^{2}$ or $A G$ ?

To be more precise, let us distinguish three types of rigidity questions according to the regularity hypothesis:

Q 1: $C^{\infty}$-rigidity. Is (up to finite covers) a $C^{\infty}$ codimension one foliation on a closed 3-manifold, which is lightlike geodesic for some Lorentz $C^{\infty}$ metric, $C^{\infty}$-diffeomorphic to the orbit foliation of a locally free $C^{\infty}$ action of $\mathbf{R}^{2}$ or $A G$ ?

Q 2: strong $C^{\infty}$-rigidity. Is (up to finite covers) a $C^{0}$ codimension one foliation on a closed 3-manifold, which is lightlike geodesic for some Lorentz $C^{\infty}$ metric, $C^{\infty}$-diffeomorphic to the orbit foliation of a locally free $C^{\infty}$ action of $\mathbf{R}^{2}$ or $A G$ ?

Q 3: $C^{0}$-rigidity. Is (up to finite covers) a $C^{0}$ codimension one foliation on a closed 3-manifold, which is lightlike geodesic for some Lorentz $C^{1}$ metric, $C^{0}$ homeomorphic to the orbit foliation of a locally free $C^{0}$ action of $\mathbf{R}^{2}$ or $A G$ ?

Some remarks are in order:

i) We always assume the metrics are at least $C^{1}$, which allows us to consider Levi-Civita connections.

ii) Of course one may combine the questions above by imposing and asking a different degree of regularity on the data.

iii) The foliations determined by actions of $\mathbf{R}^{2}$ are classified [RRW], but those determined by actions of $A G$ are completely understood only when they preserve smooth measures [Gh2].

\subsection{Regularity}

The question $\mathbf{Q} \mathbf{2}$ above asks in particular if the $C^{\infty}$ regularity of the metric does propagate to a regularity of the foliation itself. As a justification, we have the two following local regularity results:

Theorem 4. A codimension one geodesic foliation on a manifold (of any dimension) endowed with a smooth connection (e.g. a pseudo-Riemannian metric) is locally Lipschitz.

We also have the following amazing fact (see [Ze) for similar results):

Proposition 5. Let $\mathcal{F}$ be a lightlike geodesic foliation in a real analytic Lorentz 3-manifold $M$. Then $\mathcal{F}$ is analytic in some open set, unless $M$ has constant curvature.

\subsection{An easy criterion}

We are now going to give some partial answers to the rigidity questions above. We start with the following geodesibility criterion (in dimension 3).

Proposition 6. Let $M^{3}$ be a Lorentz manifold and $\mathcal{F}$ a foliation by lightlike leaves with normal foliation $\mathcal{N}$. Then $\mathcal{F}$ is geodesic if and only if there is a unit vector 
field tangent to $\mathcal{F}$ and preserving $\mathcal{N}$ (in fact in this case, any other unit vector field tangent to $\mathcal{F}$ preserves $\mathcal{N}$ ). An analogous criterion is valid for geodesibility.

Proof. Let $g$ be the induced degenerate Riemannian metric on $T \mathcal{F}$, and $Y$ a unit vector field $(g(Y, Y)=1)$ tangent to $\mathcal{F}$. The proposition follows from the following claim: $\mathcal{N}$ preserves $g$ if and only if the (local) flow of $Y$ preserves $\mathcal{N}$.

This claim is one of the ridumentary facts of the theory of geodesic and Riemannian foliations $[\mathrm{Mol}]$, and may be proved by considering the semi-distance generated by the degenerate Riemannian metric. The distance along the orbits of the flow of $Y$ coincides with the parameter arc-length. On the other hand, $\mathcal{N}$ preserves $g$ precisely if the distance between any two leaves, measured along any two $Y$-orbits cutting them, is the same.

2.2.1. A rigidity result in a dynamical context. Let $\left(M^{3}, \phi^{t}\right)$ be an Anosov flow, with infinitesimal generator $X$. Assume that $\phi^{t}$ preserves a Lorentz metric $<,>$, with some degree of regularity. By invariance and hyperbolicity, the (strong) stable bundle must be lightlike and orthogonal to $X$. In particular $X$ cannot be lightlike, and so has a constant length (by ergodicity), say 1 . By the proposition above, the weak stable foliation is lightlike geodesic, with the strong stable foliation as normal foliation.

Note that, as we shall see in the more general situation of the theorem below, the weak stable foliation is at least as regular as the metric (we assume as usual that $X$ is $C^{\infty}$ ). There are two extremal possibilities:

i) By $[\mathrm{Kan}]$ and $[\mathrm{H}-\mathrm{K}]$, a volume preserving Anosov flow on a compact 3manifold (e.g. the geodesic flow on the unit tangent bundle of a surface of negative curvature) preserves a $C^{1}$ Lorentz metric. In fact, both this metric and the weak stable foliation are $C^{1+Z y g m u n d}$ in this case. By [Mar], $\mathbf{Q} 3$ is satisfied in this case. That is, the foliation is homeomorphic to a $C^{0}$ action of $A G$. But there is no hope at this regularity level of the metric, to get more regular (for example $C^{1}$ ) actions.

ii) By [Ze1], Anosov flows preserving $C^{k}, k \geq 2$, Lorentz metrics correspond to "geometric deformations" of algebraic Anosov flows. In particular one may verify the validity of $\mathbf{Q} \mathbf{1}$ in this case, when replacing $C^{\infty}$ by $C^{k}$.

But, what about metrics, making the weak stable foliation lightlike geodesic, but are not (necessarily) invariant by the Anosov flow ? Such metrics exist even in the algebraic case, since we have some freedom to change a metric making a foliation geodesic. The following is our first verification of $\mathbf{Q} \mathbf{2}$ :

Theorem 7. Assume that the weak stable foliation of an Anosov flow on a compact 3-manifold, is lightlike geodesic for some $C^{\infty}$ metric (not necessarily invariant by the Anosov flow). Then it is $C^{\infty}$ diffeomorphic to an analytic action of $A G$. More precisely, up to finite covers, the foliation is $C^{\infty}$ diffeomorphic to the weak stable foliation of an algebraic Anosov flow. 
2.2.2. A rigidity result in a geometric context. We are now going to consider Q 2 in a particular geometrical context, that is, for manifolds of constant curvature. The nicest examples of lightlike geodesic foliations on such manifolds are surely the weak stable foliations of algebraic Anosov flows: the geodesic flows of hyperbolic surfaces and suspensions of hyperbolic linear diffeomorphisms on the 2 -torus. Their underlying spaces have constant negative and 0 curvature, respectively. They are defined by actions of $A G$. We also have examples defined by actions of $\mathbf{R}^{2}$ on flat 3 -tori and flat homogeneous nilmanifolds. The following is an affirmative answer for $\mathbf{Q} \mathbf{2}$ in this context:

Theorem 8. Let $M$ be a compact Lorentz 3-manifold of constant curvature, and $\mathcal{F}$ a $C^{0}$ lightlike geodesic foliation on it. Then, up to finite covers, $\mathcal{F}$ is $C^{\infty}$ diffeomorphic to a foliation defined by an analytic action of $\mathbf{R}^{2}$ or $A G$ (further precisions are contained in the proof).

2.2.3. General context. We also have the following two results concerning $\mathbf{Q} \mathbf{3}$, without curvature assumptions.

Theorem 9. Let $\mathcal{F}$ be a $C^{0}$ lightlike geodesic foliation in a compact Lorentz 3manifold. Suppose that $\mathcal{F}$ has no transverse invariant measure. Then $\mathcal{F}$ may be defined by a locally free $C^{0}$ action of $A G$.

We believe that (by a similar argument) this fact may be extended at least to foliations without compact leaves, but in this case we also admit actions of $\mathbf{R}^{2}$.

Theorem 10. Up to finite covers, a lightlike geodesic foliation defined by a suspension of a representation of a surface group $\Gamma=\pi_{1}(V)$ in Homeo $\left(S^{1}\right)$, is defined by a $C^{0}$ locally free action of $\mathbf{R}^{2}$ or $A G$. In fact for $V$ of genus $\geq 2$, the representation is continuously semi-conjugate to the boundary action of $\Gamma$ on the ideal boundary of $\tilde{V}$.

Finally, we have the following general qualitative result (see [God] for definitions)

Theorem 11. Let $\mathcal{F}$ be a $C^{0}$ lightlike geodesic foliation in a compact Lorentz 3-manifold. Then:

i) A leaf of $\mathcal{F}$ is homeomorphic to a plane, a cylinder or a torus.

ii) $\mathcal{F}$ has no vanishing cycles.

iii) The universal cover is homeomorphic to $\mathbf{R}^{3}$, foliated by planes.

Remark 12. The no vanishing cycles property is not valid for dimension $>3$. Indeed, the suspension (as defined in $\$ 1$ ) by the identity map, of any foliation having vanishing cycles on a 3-manifold, is a lighlike geodesible codimension 1 
foliation on a 4-manifold, which must have vanishing cycles.

Remark 13. There are foliations of compact 3-manifolds, satisfying the condition of the last proposition, but not lightlike geodesible, and therefore not defined by locally free actions of Lie groups.

In fact there are examples of foliations $\mathcal{F}$ by planes and cylinders, with no vanishing cycles, such that the tangent bundle $T \mathcal{F}$ has no non-vanishing section (even after passing to finite covers). Indeed, consider the foliation $\mathcal{F}$ defined by a representation: $h: \Gamma \in P S L(2, \mathbf{R})$ of the fundamental group of a surface $V$ of genus $\geq 2$. One may verify the two following facts. Firstly, if $h$ is injective, then all the leaves of $\mathcal{F}$ are planes or cylinders. Secondly, if $h$ is homotopic to the trivial representation $\Gamma \rightarrow 1$, then $T \mathcal{F}$ has no non-vanishing section (since otherwise, the tangent bundle of the surface $V$ would have such a section). To get examples of foliations as claimed, one observes that indeed, there are injective representations near the trivial one (a proof of this fact may be extracted from [Gol]).

I have learned these last examples and some other facts in this paper from $E$. Ghys.

\section{Proof of the regularity results}

Proof of Theorem 4. Let $M$ be a manifold endowed with a connection $\nabla$. The result of Theorem 4 is local, so we may assume that $M$ is a convex neighborhood $V$ of a fixed point $x_{0}$ (two points in $V$ are joined by a unique geodesic in $V$ ). The foliation $\mathcal{F}$ is trivial in $V$ in the sense that any leaf equals the convex hull of its intersection with the boundary $\partial V$.

In the following, we shall measure angles and distances by means of an auxiliary smooth Riemannian metric (which determines a norm $\|$. $\|$ and a distance $d$ ), but geodesics and parallel transport by means of the given connection $\nabla$.

Lemma 14. Let $V$ be a sufficiently small convex neighborhood of $x_{0}$ and $F$ a codimension 1 "relatively complete" geodesic submanifold (i.e. F equals the convex hull of $F \cap \partial V$ ).

Let $\gamma$ be a smooth curve in $V$, transverse to $F$, with $\gamma(0)=x$, and let $P_{t}$ denote the parallel transport along $\gamma$ of $P_{0}=T_{x} F$.

Then, in order that an unoriented geodesic $g$ at $\gamma(t)$, has no intersection with $F \cap V$, the angle $\angle\left(g, P_{t}\right)$ (at $\left.\gamma(t)\right)$ must satisfy:

$$
\angle\left(g, P_{t}\right) \leq c d\left(x_{0}, \gamma(t)\right),
$$

for some constant $c$, related to the size of $V$ and the $C^{1}$ size of the curve $\gamma$ (for example if $\gamma$ is taken to be a geodesic of $\nabla$, then $c$ is related to the size of $V$ and $\left.\angle\left(\gamma^{\prime}(0), P_{0}\right)\right)$. 
Proof of the lemma. For $x \in M$, let $\exp _{x}$ denote the exponential map at $x$, defined in a neighborhood $V_{x}$ of 0 in $T_{x} M$, which is mapped diffeomorphically onto $\bar{V}$. We assume that $\partial V$ is regular.

Consider $B_{x}=\exp _{x}^{-1}(\partial F) \subset T_{x} M$, where $\partial F=F \cap \partial V$, and let $C B_{x}$ be the cone in the unit ball of $T_{x} M$ determined by $B_{x}$,

$$
C B_{x}=\left\{s u, u \in B_{x}, s \geq 0,\|s u\| \leq 1\right\}
$$

Since, $\exp _{x}: V_{x} \rightarrow V$ is diffeomorphic, $C B_{x}$ delimits a region $C B_{x}^{-}$in $V_{x}$, characterized by, $u \in C B_{x}^{-}$, iff for some $s \geq 1$, $\exp _{x} s u$ belongs to $F$.

Let $g$ be an unoriented geodesic through $x$, say it is the image of $\exp _{x} s u$, $s \in[-b, a]$, with $-b u$ and $a u \in \partial V_{x}$ (that is, $\exp _{x}-b u$ and $\left.\exp _{x} a u \in \partial V\right)$.

If $g$ doesn't cut $F \cap V$, then there is no real multiple (positive or negative) $s u$ which belongs to $C B_{x}^{-}$, that is, $u \notin C B_{x}^{-} \cup-C B_{x}^{-}$.

Note that by convexity, $C B_{x} \cap-C B_{x}=0$. The complement set of $C B_{x}^{-} \cup-C B_{x}^{-}$ in the unit sphere of $T_{x} M$ is an annulus.

Consider $U$ a small neighborhood of $x_{0}$ far away from $\partial V$. Then, the map $x \in U \rightarrow C B_{x}$ is smooth in a natural way. More precisely, there is a smooth family of diffeomorphisms $\left\{f_{x}\right\}_{x \in U}$, such that $C B_{x}=f_{x}\left(C B_{x_{0}}\right)$. In particular the map $x \in U \rightarrow C B_{x}$ is Lipschitz, when the subsets of $T M$ are endowed with the Hausdorff distance $d_{H}$. We have in particular an inequality

$$
d_{H}\left(C B_{x}, C B_{x_{0}}\right) \leq(c / 2) d\left(x, x_{0}\right),
$$

for some $c$. The same is true for $C B_{x}$ replaced by $-C B_{x}$.

Now, consider the hypersurfaces $F_{t}=\exp _{\gamma(t)}\left(P_{t}\right)$, and denote by $P_{t}^{1}$ the trace of $P_{t}$ on the unit sphere of $T_{\gamma(t)} M$. A standard analysis (i.e. by using the implicit function theorem) shows that the family of hypersurfaces $\left\{F_{t}\right\}$ foliates a neighborhood of $F\left(=F_{0}\right)$ (related to the $C^{1}$ size of $\gamma$ ). Therefore $P_{t}^{1}$ is contained in the annulus determined by $C B_{\gamma(t)}$ and $-C B_{\gamma(t)}$, and in particular: $d_{H}\left(C B_{\gamma(t)}, P_{t}\right) \leq d_{H}\left(C B_{\gamma(t)},-C B_{\gamma(t)}\right)$.

It then follows that:

$$
\begin{aligned}
d_{H}\left(C B_{\gamma(t)}, P_{t}\right) & \leq d_{H}\left(C B_{\gamma(t)},-C B_{\gamma(t)}\right) \\
& \leq d_{H}\left(C B_{\gamma(t)}, P_{0}\right)+d_{H}\left(P_{0},-C B_{\gamma(t)}\right) \leq c d\left(x_{0}, \gamma(t)\right.
\end{aligned}
$$

Also, for any vector $u$ in the annulus delimited by $C B_{\gamma(t)}$ and $-C B_{\gamma(t)}$, we have:

$$
\angle\left(u, P_{t}\right) \leq d_{H}\left(C B_{\gamma(t)},-C B_{\gamma(t)}\right) \leq c d\left(x_{0}, \gamma(t)\right) .
$$

Note that the parallel transport with respect to $\nabla$ was just introduced for aesthetic reasons. One may as well use local charts and Euclidean parallel transport there. 
The lemma implies Theorem 4. Indeed, for a foliation $\mathcal{F}$, we may let $x_{0}$ be any point of the curve $\gamma$. Therefore, in a Euclidean chart, we get: $\angle\left(T_{x} \mathcal{F}_{x}, T_{y} \mathcal{F}_{y}\right) \leq$ $c d(x, y)$, where $x$ and $y$ are points of $\gamma$ (the angle is Euclidean). This means that $\mathcal{F}$ is locally Lipschitz.

Note that we have in fact proved:

Proposition 15. Let $\mathcal{F}$ be a partition of a subset $A \subset V$ into relatively complete geodesic codimension 1 submanifolds. Then $\mathcal{F}$ is locally Lipschitz (in particular it can be extended to the closure of $A$ )

Proof of Proposition 5. Let $M$ be a pseudo-Riemannian manifold. On the Grassmann bundle $G r^{d}(M) \rightarrow M$, there is a tautological geodesic d-plane field. At a point $(x, P) \in G r^{d}(M)$, this plane field is the horizontal lift (with respect to the Levi-Civita connection) of $P$ itself (see [Ze3] for further details). An integral submanifold through $(x, P)$ corresponds to a totally geodesic submanifold tangent to $P$.

A classical theorem due to Schur states that, for $1<d<\operatorname{dim}(M)$, the tautological plane field is integrable if and only if $M$ has constant curvature.

For Lorentz manifolds, the tautological plane field is tangent to the sub-Grassmann $G r_{0}^{n-1}(M)$ of lightlike hyperplanes.

Lemma 16. For a Lorentz 3-manifold $M$, the restriction on $G r_{0}^{2}(M)$ of the tautological plane field is integrable, if and only if $M$ has constant curvature.

Proof. Fix $x \in M$ and let $X \in T_{x} M$ be a unit (spacelike) vector. The metric on the orthogonal $X^{\perp}$ is Lorentzian and so has exactly two lightlike directions oriented by two vectors $Y$ and $Z$. For vectors $A_{1}, \ldots, A_{d}$, we denote by $\operatorname{Span}\left\{A_{1}, \ldots, A_{d}\right\}$ the vector space that they generate. By hypothesis $\operatorname{Span}\{X, Y\}$ determines a totally geodesic surface. In particular the curvature $A_{X}(Y)=R(Y, X) X \in \operatorname{Span}\{X, Y\}$, and hence is collinear to $Y$, since it is orthogonal to $X$. Therefore $A_{X}(Y)=$ $\alpha(X, Y) Y$, for some real $\alpha(X, Y)$. In the same way $A_{X}(Z)=\alpha(X, Z) Z$. But:

$$
<R(Y, X) X, Z>=\alpha(X, Y)<Y, Z>=<R(Z, X) X, Y>=\alpha(X, Z)<Y, Z>
$$

We have $\langle Y, Z>\neq 0$, since otherwise the metric would be degenerate. Therefore $\alpha(X, Y)=\alpha(X, Z)$, and so on $X^{\perp}, A_{X}$ is just a multiplication by a scalar $\alpha_{X}$.

Now if $X^{\prime}$ is another unit vector orthogonal to $X$, then: $\left.<R\left(X^{\prime}, X\right) X, X^{\prime}\right\rangle=$ $\alpha_{X}$. The same argument applied to $X^{\prime}$ leads to an equality with $\alpha_{X^{\prime}}$. In a standard way we deduce from this that the sectional curvatures of all non-lightlike planes equal a constant $\alpha=\alpha(x)$. By Schur's lemma, this does not depend on $x$ and $M$ has constant curvature $\alpha$.

If $\operatorname{dim}(M)=3$, then $\operatorname{dim} G r_{0}^{2}(M)=4$. If $\mathcal{F}$ is a lightlike geodesic foliation then $\operatorname{Gr}(\mathcal{F})$, the image of the map, $x \in M \rightarrow T_{x} \mathcal{F}_{x}$, is a topological 3-manifold. 
If the metric is analytic, the integrability domain $\mathcal{I}$ of the tautological geodesic plane field on $G r_{0}^{2}(M)$ (which may be defined naturally, see [Ze]) is an analytic set .

Therefore, $\mathcal{I}$ equals all $G r_{0}^{2}(M)$ whenever it has dimension 4 . If not, $\operatorname{dim} \mathcal{I}=3$ since $\mathcal{I}$ contains $\operatorname{Gr}(\mathcal{F})$. Therefore, $\operatorname{Gr}(\mathcal{F})$ is a topological 3-manifold in a 3dimensional analytic set.

In particular, near each point $x \in G r(\mathcal{F})$ which is a regular point for $\mathcal{I}, G r(\mathcal{F})$ is an analytic submanifold. Now, in general, if the image of a section of a bundle is an analytic submanifold, then away from the set of points where this submanifold is tangent to the vertical, the section is analytic. But the set of vertical points has at least codimension 1. This proves (a strong version of) Proposition 5.

\section{Weak stable foliations of Anosov flows: Proof of Theorem 7}

By a result of E. Ghys [Gh3], the weak stable foliation of an Anosov flow on a 3 -manifold, is $C^{\infty}$-diffeomorphic to that of an Algebraic flow, iff, it is $C^{\infty}$.

Therefore Theorem 7 reduces to the fact that, if the weak stable foliation of an Anosov flow is lightlike geodesic for some $C^{\infty}$ Lorentz metric, then this foliation is $C^{\infty}$. It is worth saying that weak stable foliations of algebraic Anosov flows are (explicitly) parameterized by actions of $A G$. The same is true for foliations diffeomorphic to them.

Let $\phi^{t}$ be an Anosov flow, $X$ its infinitesimal generator, $W^{s}$ its weak stable foliation, $W^{u}$ its weak unstable foliation and $\mathcal{N}$ the normal foliation of $W^{s}$.

Obviously, there is no reason for $\mathcal{N}$ to be the strong stable foliation of $\phi^{t}$. In fact the difficulty in the proof lies in the possible existence of tangency points of $X$ and $\mathcal{N}$.

Since the metric on $T W^{s}$ is positive (non-definite), we have everywhere: < $X, X>\geq 0$. Let $S$ be the open set $\{x \in M /<X(x), X(x) \neq 0\}$ and $L=\{x \in$ $M /<X(x), X(x)>=0\}$ its complementary set .

Let's show that $\mathcal{N}$ is $C^{\infty}$ on $S$. The same will be true for $T W^{s}$ since it is just the orthogonal distribution of $\mathcal{N}$. For $x \in S$, the orthogonal $X^{\perp}(x)$ is timelike, i.e. the restriction to it of $\langle$,$\rangle is Lorentzian. Thus, since \operatorname{dim} X^{\perp}=2$, there are exactly two isotropic directions in $X^{\perp}(x)$. Therefore, they are (like $X$ ) $C^{\infty}$. Obviously, $\mathcal{N}$ is one of these directions, and is therefore $C^{\infty}$.

By the same argument, $\mathcal{N}$ is $C^{\infty}$ in the interior of $L$.

Consider $D$ the set of points having a neighborhood in which $\mathcal{N}$ (or equivalently $\left.W^{s}\right)$ is $C^{\infty}$. It is open, and dense by what precedes. Theorem 7 means nothing but that $D=M$.

Observe that $D$ is invariant by $\phi^{t}$ (since $\phi^{t}$ is $C^{\infty}$ and preserves $W^{s}$ ). Observe also that if $x \notin D$, then all its $\phi^{t}$-orbit is lightlike, in fact, it coincides with its $\mathcal{N}$-orbit.

Consider the set $M-D$. It is closed, and along it, $\phi^{t}$ and $\mathcal{N}$ orbits coincide. 
This implies that $M-D$ cuts any weak stable leaf, at most along one $\phi^{t}$-orbit. Indeed, if two orbits are contained in the same weak stable leaf, then, as being leaves of $\mathcal{N}$ (which is transversally Riemannian along the weak stable foliation), these orbits are "parallel", which contradicts the contracting character of $\phi^{t}$ along $W^{s}$.

Recall now that there is a spectral decomposition of $M$ into basic sets in which $\phi^{t}$ is topologically transitive [Sma]. From the above fact, that is, $M-D$ contains at most one $\phi^{t}$-orbit from any $W^{s}$-leaf, one sees that $M-D$ cannot (fully) contain a "non-trivial" basic set, that is one which is not reduced to an (isolated) periodic orbit.

Therefore, since periodic orbits are dense in basic sets, $D$ contains periodic orbits from each non-trivial basic set. In fact, since $M-D$ is closed, $D$ contains all the weak unstable leaves of such orbits.

Fact 17. Let $\Lambda_{1}, \ldots, \Lambda_{d}$ be the non-trivial (i.e. not reduced to periodic orbits) basic sets of an Anosov flow $\left(M, \phi^{t}\right)$ and $x_{i} \in \Lambda_{i}$ (periodic or not). Then the union $\bigcup_{i=1}^{i=d} W^{u}\left(x_{i}\right)$ is dense in $M$.

Proof. Let $W^{u}\left(\Lambda_{i}\right)=\bigcup_{x \in \Lambda_{i}} W^{u}(x)$. By continuity of $W^{u}$ and since $W^{u}\left(x_{i}\right) \cap \Lambda_{i}$ is dense in $\Lambda_{i}$, we have that $W^{u}\left(x_{i}\right)$ is dense in $W^{u}\left(\Lambda_{i}\right)$. Therefore $\bigcup_{i=1}^{i=d} W^{u}\left(x_{i}\right)$ is dense in $A=\bigcup_{i=1}^{i=d} W^{u}\left(\Lambda_{i}\right)$.

To finish the proof it suffices to show that $A$ is dense in $M$. Let $\Lambda_{d+1}, \ldots \Lambda_{m}$ be the trivial basic sets. Then $M=\bigcup_{i=d+1}^{i=m} W^{u}\left(\Lambda_{i}\right) \cup A$. But for $i>d$, for some $x_{i}$, $W^{u}\left(\Lambda_{i}\right)=W^{u}\left(x_{i}\right)$, and hence, this is an immersed submanifold of codimension $>0$ (notice that $\phi^{t}$ is an Anosov flow and not just Axiom-A). It follows that $A$ is dense in $M$.

To finish the proof of Theorem 7 , that is, $D=M$, we would like to apply the remarkable result of [F-L], which says that, if the strong stable distribution is $C^{\infty}$ along a dense (in $M$ ) weak unstable leaf, then the strong stable foliation is everywhere $C^{\infty}$. In the non-necessarily transitive case, the same proof yields the following generalization:

Lemma 18. Assume that for a finite subset of periodic points $x_{1}, \ldots, x_{d}$, the union $\cup W^{u}\left(x_{i}\right)$ is dense in $M$, and that the weak stable distribution is $C^{\infty}$ along each $W^{u}\left(x_{i}\right)$. Then the weak stable foliation is $C^{\infty}$ everywhere.

From the previous discussion, $D$ contains such a finite set of periodic orbits. This completes the proof of Theorem 7 . 


\section{Lorentz manifolds of constant curvature: Proof of Theorem 8}

From [Car] and [Kli], compact Lorentz manifolds of constant curvature are complete. The study of foliations on such manifolds, is thus equivalent to that of foliations on their universal covers which are furthermore invariant by the action of the fundamental group.

Firstly, compact Lorentz manifolds of constant positive curvature do no exist [C-M]. It remains to consider the non-positively case.

The flat case. Let $M$ be a compact flat Lorentz 3-manifold. By [F-G], up to finite covers, $\Gamma=\pi_{1}(M)$ is contained in a connected solvable group $H$ acting isometricaly and simply transitively on the Minkowski space $\mathbf{R}^{2,1}$, called the crystalographic hull of $\Gamma$. It is contained (maybe properly) in the algebraic closure of $\Gamma$.

A geodesic foliation on $M$ lifts to a foliation of $\mathbf{R}^{2,1}$ by parallel hyperplanes, since it is a (global) foliation of $\mathbf{R}^{2+1}$ by affine hyperplanes.

The algebraic closure, and hence also $H$ preserve this foliation. Therefore, the foliation on $M$ identified with $H \backslash \Gamma$, is determined by a subgroup $G \subset H$, acting by the left on $M=H \backslash \Gamma\left(G\right.$ is the stabilizer in $H$ of one leaf in $\left.\mathbf{R}^{2,1}\right)$.

The anti de Sitter case. In dimension 3, anti de Sitter manifolds (i.e Lorentz manifolds of constant negative curvature) are locally isometric to the Lorentz manifold $X=P S L(2, \mathbf{R})$ (the group of modular $2 \times 2$-matrices, modulo \pm identity), endowed with its Killing form.

By bi-invariance, the direct product $G=P S L(2, \mathbf{R}) \times P S L(2, \mathbf{R})$ acts isometricaly on $X$ by: $\left(\left(g_{1}, g_{2}\right), x\right) \rightarrow g_{1} x g_{2}{ }^{-1}$. One may prove (essentially by dimension arguments) that we obtain all the identity component of $\operatorname{Isom}^{+}(X)$.

A compact anti de Sitter manifold is covered by $\tilde{X}$ (since it is complete, as was said above). More precisely:

Proposition 19. ([K-R]) Let $M^{3}$ be a compact complete anti de Sitter manifold. Then up to finite covers (that is by taking a quotient of a finite covering of $M$ ), there is a surface group $\Gamma \subset P S L(2, \mathbf{R})$ and $c: \Gamma \rightarrow P S L(2, \mathbf{R})$ a homomorphism such that the holonomy group of $M$ is $\operatorname{Graph}(c)=\{(\gamma, c(\gamma)), \gamma \in \Gamma\}$ (acting freely properly discontinuously on $X$ by $\left.(\gamma, c(\gamma)) x=\gamma x c(\gamma)^{-1}\right)$.

Geodesics of $X$. By bi-invariance of the Killing form, one sees that the one parameter groups are geodesic. More generally, any geodesic is a (left or right) translation of a one parameter group. This fact extends to lightlike geodesic hypersurfaces: they are of the form $x H$ (or $H x$ ), where $x \in P S L(2, \mathbf{R}$ ), and $H$ is a parabolic subgroup, that is, $H$ is conjugate to the affine group $A G$, identified with the group of upper triangular unimodular $2 \times 2$-matrices.

Fact 20. Two lightlike geodesic submanifolds $A=A G$ and $B=\left(g A G g^{-1}\right) x$ must intersect non-trivially, unless $B=g A$. 
Proof. Let $\pi: P S l(2, \mathbf{R}) \rightarrow P S L(2, \mathbf{R}) / A G=S^{1}$ be the projection with $\pi(A G)=$ $1 \in S^{1}$. The disjointness condition means $\pi\left(g A G g^{-1}\right) x$ does not contain 1 , or in other words, the orbit of $\pi(x)$ under $g A G g^{-1}$ does not contain 1. But $g A G g^{-1}$ acts on $S^{1}$ as a parabolic group with one fixed point $g(1)$. Hence 1 is not in the orbit of $\pi(x)$ iff $\pi(x)=g(1) \neq 1$. Thus $\left(g A G g^{-1}\right) x=g A G\left(g^{-1} x\right)=g A G$.

We deduce from this fact that, up to isometry, a lightlike geodesic foliation of $X$ is given by a right action of $A G$, i.e. the leaves are $\{g A G, g \in P S L(2, \mathbf{R})\}$. The isotropy group of this foliation (not the action) is $P S L(2, \mathbf{R}) \times A G$. The space of leaves is $X / A G=S^{1}$ and the action of $P S L(2, \mathbf{R}) \times A G$ on it, factors through the usual projective action of $P S L(2, \mathbf{R})$ on $S^{1}$.

The (global) holonomy group of our foliation $(M, \mathcal{F})$ is canonically equivalent to the action of $\Gamma \subset P S L(2, \mathbf{R})$ on $S^{1}$. It is the same as that of the weak stable foliation of the geodesic flow of the surface $V=\Gamma \backslash P S L(2, \mathbf{R}) / S O(2)$, which is the orbit foliation of the right action of $A G$ on $\Gamma \backslash P S L(2, \mathbf{R})$.

This later foliation is also defined by the suspension of the inclusion representation of $\Gamma=\pi_{1}(V) \rightarrow P S L(2, \mathbf{R}) \subset$ Homeo $\left(S^{1}\right)$.

Now, we prove that the foliation $\mathcal{F}$ is $C^{\infty}$-conjugate to the weak stable foliation (of the geodesic flow), by showing that also $\mathcal{F}$ is defined as a suspension of the same representation, that is, up to a $C^{\infty}$ isotopy $\mathcal{F}$ is transverse to the circle fibration of the unit tangent bundle: $T^{1} V=\Gamma \backslash P S L(2, \mathbf{R}) \rightarrow V$.

By [Lev], this will be the case whenever we prove that $\mathcal{F}$ has no compact leaves. Following the notations of the above fact, an element $(\gamma, c(\gamma))$ belongs to $S_{g A G}$, the stabilizer of a leaf $g A G$, if and only if $\gamma$ stabilizes $\pi(g) \in S^{1}$. By compactness of $V$, the stabilizer in $\Gamma$ of a point in $S^{1}$ is cyclic (may be trivial). Therefore the leaves of $\mathcal{F}$ are all cylinders or planes. $\diamond$

\section{Qualitative results: Proof of Theorems 9, 10 and 11}

Proof of Theorem 9. Let $M$ be a compact Lorentz 3-manifold endowed with a lightlike geodesic foliation $\mathcal{F}$, and $\mathcal{N}$ its normal foliation. Choose $X$ a nonvanishing vector field orienting $\mathcal{N}$ (remember that we have assumed everything orientable), and let $Y$ be a unit vector field (that is $\langle Y(x), Y(x)\rangle=1$, for all $x$ ) tangent to $\mathcal{F}$.

Consider on $T \mathcal{F}$ the Riemannian metric $h$ for which, $X$ and $Y$ are orthogonal and both of them have length 1 .

Call $\mathcal{G}$ the foliation determined by $Y$. The duality between Riemannian and geodesic foliations (see for instance [Mol]) says that the leaves of $\mathcal{G}$ are geodesic in any Riemannian leaf $(F, h)$ of $\mathcal{F}$.

Lemma 21. Let $\tilde{\mathcal{G}}$ be a geodesic foliation on a simply connected complete Riemannian 2-manifold $(\tilde{F}, \tilde{h})$. Then the leaves of $\tilde{\mathcal{G}}$ are minimizing. 
Proof. We argue by contradiction. For a point $x$ in a leaf $\tilde{\mathcal{G}_{0}}$, let $y$ be the first point (in the sense of some orientation) in that leaf, which may be joined by another geodesic segment $l$. Then $l$ is transverse to $\tilde{\mathcal{G}}$, since if it is somewhere tangent to a leaf, it would be contained in it. Therefore the foliation $\tilde{\mathcal{G}}$ restricted to the disc $\Delta$ delimited by $\tilde{\mathcal{G}_{0}}$ and $l$, is tangent to a part of $\partial \Delta$ (i.e. an interval) and transverse to the other part. This is impossible.

Since by hypothesis $\mathcal{F}$ has no transverse measure, Candel's Theorem [Can] implies that it is uniformizable, that is, there is a $C^{0}$ Riemannian metric $h_{0}$ on $T \mathcal{F}$ such that any leaf $\left(F, h_{0}\right)$ is hyperbolic, i.e. of constant curvature -1 .

By compactness, the metrics $h$ and $h_{0}$ are equivalent. In particular, by the lemma above, for any leaf $F$, the leaves of $\tilde{\mathcal{G}}$ in its universal cover $\left(\tilde{F}, \tilde{h_{0}}\right)$, which is a hyperbolic plane, are (uniformly) quasi-geodesic (see [Gr2] for definitions). The proof of Theorem 9 will follow from the following general fact.

Theorem 22. Let $M$ be a topological space endowed with a hyperbolic 2-dimensional lamination $\mathcal{F}$, that is, there is a continuous Riemannian metric $h_{0}$ on $T \mathcal{F}$, determining a hyperbolic metric on leaves.

Suppose that $\mathcal{F}$ has a one dimensional sub-lamination $\mathcal{G}$, (that is, leaves of $\mathcal{G}$ are contained in leaves of $\mathcal{F})$. Suppose that on each leaf of $\mathcal{F}, \mathcal{G}$ determines a foliation (i.e. with full support in that leaf) with uniformly quasi-geodesic leaves (the quasi-geodesic constants are uniform on $M$ ).

Then $\mathcal{F}$ may be parameterized by an action of $A G$.

Proof. First, let's work on with an individual leaf $F$ of $\mathcal{F}$ (in other words $M$ is reduced to one leaf). It is a hyperbolic surface endowed with a 1-dimensional quasi-geodesic foliation. Lift it to the universal cover $\tilde{F}$, which is a hyperbolic plane.

In a hyperbolic plane, a quasi-geodesic foliation can be canonically homotoped to a geodesic one. This follows by applying the canonical straighting process (see [Gr2], §7.1) which sends a quasi-geodesic to its asymptotic geodesic.

It is standard that this yields a geodesic lamination (that is, the obtained geodesics do not intersect).

On the other hand, the straighting homotopy translates points by a bounded distance, and thus extends continuously, as the identity map on the ideal boundary of $\tilde{F}$. This homotopy is in particular surjective. Therefore, we get a geodesic foliation (i.e. a lamination with full support) on $\tilde{F}$.

Since this homotopy is natural, it descends to a homotopy on $F$ itself, sending the initial quasi-geodesic foliation to a geodesic one.

In fact, applying this construction individually to $\mathcal{F}$-leaves, we get a leafwise homotopy on $M$, that is, $f: M \rightarrow M$, preserving each leaf of $\mathcal{F}$ and sending $\mathcal{G}$ to a geodesic sub-foliation $\mathcal{H}$.

We have now to check that $f$ is continuous on $M$, or equivalently that $\mathcal{H}$ is 
continuous on $M$.

For this sake, recall the following characterization of $f$ : for any leaf $\mathcal{G}_{x}, f\left(\mathcal{G}_{x}\right)$ is the unique geodesic in $\mathcal{F}_{x}$ having a finite Hausdorff distance from $\mathcal{G}_{x}$ (in the leaf $\left.\mathcal{F}_{x}\right)$.

Let $\left(x_{n}\right)$ be a sequence of points of $M$ converging to $x \in M$. We may suppose that $f\left(\mathcal{G}_{x_{n}}\right)$ converges to a geodesic $\mathcal{H}_{0}$ in $\mathcal{F}_{x}$. To show that $\mathcal{H}_{0}$ equals $f\left(\mathcal{G}_{x}\right)$, we use the above characterization of $f$. However, instead of $\mathcal{G}$-leaves, we must take long but compact pieces of them, and instead of geodesics, we take segments of geodesics. By continuity of the Riemannian metric on $T \mathcal{F}$, and continuity of $\mathcal{F}$, we deduce that, big pieces of $\mathcal{H}_{0}$ are at a finite distance from big pieces of $\mathcal{G}_{x}$ and hence also from big pieces of $f\left(\mathcal{H}_{x}\right)$. This implies that $f\left(\mathcal{H}_{x}\right)=\mathcal{H}_{0}$.

Let's return to the individual leaf $F$ which is now endowed with a geodesic foliation.

Via the Klein model, this foliation on $\tilde{F}$, is just a foliation by straight segments of the unit euclidean 2-ball $B$. Let's show that such a foliation determines a set of 1 or 2 points in the boundary $S^{1}$ of this ball. Indeed, by obvious topological and dynamical triviality, the quotient space of the foliation is $\mathbf{R}$, and the leaves are the fibers of a trivial topological fibration $B \rightarrow \mathbf{R}$. Let $t \in \mathbf{R} \rightarrow c(t) \in B$ be a section of this foliation, that is a complete transversal of the foliation. Suppose that $c(0)$ passes through the leaf of the center 0 of $B$. Let $l(t)$ be the Euclidean length of the leaf of $c(t)$. It is easy to see that $l$ is decreasing on each interval $[0, \infty[$ and ]$-\infty, 0$ ] (one firstly shows that $l$ is locally decreasing). Therefore, when $t \rightarrow \infty$ (resp. $-\infty$ ), the leaf of $c(t)$ tends to a point in the boundary of $B$, which gives the promised set of 1 or 2 points.

If everything is orientable, then we can naturally choose one of these points.

Now, a point at infinity of a hyperbolic plane, gives rise to a canonical action of $A G$. Indeed, $A G$ is isomorphic to the stabilizer of a point at infinity in the isometry group of a hyperbolic plane.

This action goes down to $F$. (Note here that $F$ must have an elementary fundamental group, since it respects a set of 1 or 2 points at infinity).

This construction applies to all leaves of $\mathcal{F}$ in $M$, and we thus get a parameterization of $\mathcal{F}$ by $A G$.

As above, it remains to show that the $A G$-action is continuous. As above, the idea is to find an interesting characterization of the set of 1 or 2 points associated to a foliation by geodesic segments on an Euclidean 2-ball. Here follows such a characterization which allows us to complete the proof of the theorem. Let $x$ be a point of the ball $B$, and $D$ a connected foliated region delimited by the $x$-leaf and transversally compact (i.e. it contains a compact set of leaves).

Let $y$ be a point in the small component of $B-D$ (that is one which does not contain $x$ in its closure). Consider the geodesic segment $[x, y]$. Then, when $D$ becomes large, the segment $[x, y]$ converges to a semi-geodesic emanating from $x$ and ending at one of the points at infinity determined by the foliation. 
Proof of Theorem 11. The fact that a complete surface $F$ admitting a Riemannian foliation is homeomorphic to a plane, a cylinder or a torus, may be deduced from the general theory of [Gh1] (see also a direct proof in [Ze1]). The idea is the following. Let $(F, \mathcal{N})$ be such a Riemannian foliated 2-manifold. In the universal cover, $\tilde{\mathcal{N}}$ is defined by a Riemannian fibration $d: \tilde{F} \rightarrow \mathbf{R}$, where $\mathbf{R}$ is endowed with a complete (Euclidean) metric. Furthermore $d$ is equivariant with respect to the holonomy representation $h: \pi_{1}(F) \rightarrow I \operatorname{som}^{+}(\mathbf{R})=\mathbf{R}$ (we assume everything orientable). Consideration of $\operatorname{Ker}(h)$ allows one to see that $\pi_{1}(F)$ cannot be free with more than one generator. Therefore, if $F$ is an open surface, it is a plane or a cylinder. On the other hand, if $F$ is closed, then it is a torus, since it is orientable and admits a non-singular foliation.

In fact, an elementary argument yields:

Fact 23. Let $(F, \mathcal{N})$ be a complete Riemannian foliated 2-manifold. Then $\mathcal{N}$ is homeomorphic to a linear foliation on: a Euclidean plane $\mathbf{R}^{2}$, a Euclidean cylinder $\mathbf{R}^{2} / \mathbf{Z}$, or a Euclidean torus $\mathbf{R}^{2} / \mathbf{Z}^{2}$. (In particular in the cylinder or torus case, if one leaf of $\mathcal{N}$ is compact then, all the leaves are).

Now, we proceed to prove that $\mathcal{F}$ has no vanishing cycles. Suppose by contradiction that there is a continuous family $\left\{l_{t}, t \in[0,1]\right\}$ of closed loops in leaves $F_{t}$, such that $l_{0}$ is not homotopically trivial in $F_{0}$ but for all $t>0, l_{t}$ is null homotopic in $F_{t}$.

In particular $F_{0}$ is a cylinder or a torus (and not a plane). The argument in the two cases is the same, so we will do it in the case of a cylinder.

To begin with, suppose that $\left(F_{0}, \mathcal{N}\right)$ has no compact leaves. Then, after homotopy, $l_{0}$ becomes transverse to $\mathcal{N}$. Thus, for $t$ near 0 , also $l_{t}$ is transverse to $\mathcal{N}$. Therefore, $\mathcal{N}$ determines a non-singular foliation on the disc delimited by $l_{t}$ in $F_{t}$, which is transverse to the boundary. Impossible!

Suppose now that all the leaves of $\left(F_{0}, \mathcal{N}\right)$ are compact. Then, after homotopy, $l_{0}$ is a leaf, and $\mathrm{s}$ in particular, it is transverse to the orthogonal foliation $\mathcal{G}$ (which was introduced in the begining of the proof of Theorem 9). This leads to the same contradiction as in the first case.

This finishes the proof of no vanishing cycles property. It implies that all the leaves of $(\tilde{M}, \tilde{\mathcal{F}})$ are planes. Therefore by Palmeira's Theorem [Pal], $\tilde{M}$ is homeomorphic to $\mathbf{R}^{3}$ foliated by planes.

Remark 24. By an unpublished theorem of Duminy, a $C^{2}$ lightlike geodesic foliation (in dimension 3) has no exceptional set. Indeed this theorem says that exceptional sets of $C^{2}$ (codimension 1) foliations have leaves with infinitely many ends. In particular, if a $C^{2}$ lightlike geodesic foliation has no compact leaves, then, it is minimal, that is all its leaves are dense.

Proof of Theorem 10. Let $\mathcal{F}$ be a lightlike geodesic foliation defined by the 
suspension of a representation $h: \Gamma=\pi_{1}(V) \rightarrow \operatorname{Homeo}\left(S^{1}\right)$, that is, $\mathcal{F}$ corresponds to the factor $\tilde{V}$ in the quotient $M=\tilde{V} \times S^{1} / \Gamma$, where $\Gamma$ acts diagonally by $\gamma(\tilde{v}, s)=(\gamma \tilde{v}, h(\gamma) s)$.

By our orientability hypothesis and Theorem $11, V$ is a torus or an orientable surface of higher genus.

In the torus case, any suspension gives rise to a locally free action of $\mathbf{R}^{2}$. Indeed, $\tilde{V}$ is then identified with $\mathbf{R}^{2}$ (as an affine space) and hence $\mathbf{R}^{2}$ acts on $\tilde{V} \times S^{1}$. This action passes to $M$.

In the case where $V$ has genus $\geq 2, \tilde{V}$ is the hyperbolic plane $\mathbf{H}^{2}$. Theorem 18 applies, and therefore, $\mathcal{F}$ is defined by a continuous action of $A G$. One sees in a standard way that this permits to select, for every leaf $\mathbf{H}^{2} \times\{s\}, s \in S^{1}$, a boundary point $u(s) \in \partial_{\infty} \mathbf{H}^{2}$. Moreover, $u: S^{1} \rightarrow \partial_{\infty} \mathbf{H}^{2}$ is continuous and equivariant: $u(h(\gamma)(s))=\gamma(u(s))$. In particular the representation $h$ is injective, and $u$ semi-conjugates the given action on $S^{1}$ (determined by $h$ ) to the boundary action of $\Gamma=\pi_{1}(V)$ on the ideal boundary $\partial_{\infty} \mathbf{H}^{2}$.

\section{References}

[A-F] S. Adams and A. Freire, Non-negatively curved leaves in foliations, J. Diff. Geom. (1991), 681-700.

[B-E] J. Beem and P. Ehrlich, Global Lorentzian geometry, Dekker, New York 1981.

[Can] A. Candel, Uniformization theorem for surface laminations, Ann. Sci. Ec. Norm. Sup. 26 (1993), 489-516.

[C-M] E. Calabi and L. Markus, Relativistic space forms, Ann. of. Math. 75 (1962), 63-76.

[Car] Y. Carrière, Autour de la conjecture de L. Markus sur les variétés affines, Invent. Math. 95 (1989), 615-628.

[D-G] G. D'Ambra and M. Gromov, Lectures on transformation groups: geometry and dynamics, Surveys in Differential Geometry (Supplement to the Journal of Differential Geometry), 1 (1991) 19-111.

[F-G] D. Fried and W. Goldman, Three dimensional affine crystallographic groups, Adv. Math. 47 (1983), 1-49.

[F-L] P. Foulon., F. Labourie, Sur les variétés compactes asymptotiquement harmoniques, Invent. Math. 109 (1992), 97-111.

[Gh1] E. Ghys, Classification des feuilletages totalement géodésiques de codimension un, Comment. Math. Helv. 58 (1983), 543-572.

[Gh2] E. Ghys, Actions localement libres du groupe affine, Invent. Math. 82 (1985), 479-526.

[Gh3] E. Ghys, Rigidité différentiable des groupes fuchsiens, Publ. Math. I.H.E.S. 78 (1993), 163-185.

[God] C. Godbillon, Feuilletages, Études géométriques, Birkhäuser 1991.

[Gol] W. M. Goldman, Topological components of spaces of representations, Invent. Math. 93 (1988), 557-607.

[Gr1] M. Gromov, Foliated Plateau problem, Geom. Func. Analysis 1 (1991), 14-79.

[Gr2] M. Gromov, Hyperbolic groups, S. Gersten (ed.), Essays in Group Theory, Springer 1987, 75-265.

[H-K] S. Hurder and A. Katok, Differentiability, rigidity and Godbillon-Vey classes for Anosov flows, Pub. I.H.E.S, $\mathbf{7 2}$ (1990), 5-61.

[Kan] M. Kanai, Geodesic flows of negatively curved manifolds with smooth stable and unstable 
foliations, Erg. Theory. Dyn. Sys. 8 (1988), 251-241.

[K-N] S. Kobayachi and K. Nomizu, Foundations of Differential Geometry, Wiley (interscience), New York, Vol I (1963).

[K-R] R. Kulkarni and F. Raymond, 3-dimensional Lorentz space- forms and Seifert fiber spaces, J. Diff. Geom. 21 (1985), 231-268.

[Kli] B. Klingler, Complétude des variétés lorentziennes à courbure constante. Math. Ann. 306 (1996), 353-370.

[Lev] G. Levitt, Feuilletages des variétés de dimension 3 qui sont des fibrés en cercles, Comment. Math. Helvetici 53 (1978), 572-594.

[Lic] A. Lichnerowicz, Théories relativistes de la gravitation et de l'électromagnétisme. Relativité générale et théories unitaires. Masson et Cie, Paris 1955.

[Mar] B. Marcus, Ergodic properties of horocyclic flows for surfaces of negative curvature, Ann. of. Math. 105 (1977), 81-105.

[Mol] P. Molino, Riemannian Foliations, Birkhüser 1988.

[O'N] B. O'Neill, Semi-Riemannian geometry, Academic press 1983.

[Pal] C.F.B. Palmeira, Open manifolds foliated by planes, Ann. Math., 107 (1978), 109-131.

$[\mathrm{P}-\mathrm{R}] \mathrm{R}$. Penrose, W. Rindler, Spinors and space-time. Vol. 2. Spinor and twistor methods in space-time geometry. Cambridge University Press, Cambridge-New York 1986.

[RRW] H. Rosenberg, R. Roussarie and D. Weil, A classification of closed orientable 3-manifolds of rank two, Ann. of Math. 91 (1970), 449-464.

[R-T] I. Robinson, A. Trautman, Cauchy-Riemann structures in optical geometry. Proceedings of the fourth Marcel Grossmann meeting on general relativity, (Rome, 1985), 317-324, North-Holland, Amsterdam-New York (1986).

[Sma] S. Smale, Differentiable dynamical systems, Bull. Amer. Math. Soc. 73 (1967), 747-817.

[Ze1] A. Zeghib, Killing fields in compact Lorentz 3-manifolds, J. Diff. Geom. 43 (1996), 859-894.

[Ze2] A. Zeghib, Isometry groups and geodesic foliations of Lorentz manifolds. Part I: Foundations of Lorentz dynamics. Preprint, available at: http://umpa.ens-lyon.fr/ ${ }^{\sim}$ zeghib/pubs.html

[Ze3] A. Zeghib, Isometry groups and geodesic foliations of Lorentz manifolds. Part II: Geometry of analytic Lorentz manifolds with large isometry groups. Preprint, available at: http://umpa.ens-lyon.fr/ zeghib/pubs.html

[Zim] R. Zimmer, Ergodic theory, semisimple Lie groups and foliations by manifolds of negative curvature, Publ. Math. IHES 55 (1982), 37-62.

Abdelghani Zeghib

CNRS, UMPA, École Normale Supérieure de Lyon

46, allée d'Italie

F-69364 Lyon cedex 07

France

e-mail: Zeghib@umpa.ens-lyon.fr

http://umpa.ens-lyon.fr/ ${ }^{\sim}$ zeghib/

(Received: October 24, 1994) 